\title{
Endobronchial ultrasound guided-transbronchial needle aspiration vs. conventional transbronchial needle aspiration in the diagnosis of mediastinal masses: A meta-analysis
}

\author{
TIANYI ZHU ${ }^{1 *}$, XINJI ZHANG ${ }^{2 *}$, JUNNAN XU $^{3}$, JUN TIAN ${ }^{4}$, HUI LI $^{2}$, \\ DAN LIU ${ }^{1}$, RUOHUA CHEN ${ }^{1}$, QIANG LI $^{1}$ and CHONG BAI ${ }^{1}$ \\ ${ }^{1}$ Department of Respiratory Medicine, Changhai Hospital; Departments of ${ }^{2}$ Health Statistics and ${ }^{3}$ Pathophysiology, \\ Second Military Medical University, Shanghai; ${ }^{4}$ Department of Immunology, \\ Zhejiang University, Hangzhou, Zhejiang, P.R. China
}

Received June 19, 2013; Accepted October 17, 2013

DOI: $10.3892 / \mathrm{mco} .2013 .206$

\begin{abstract}
Whether an endobronchial ultrasound (EBUS) is required for transbronchial needle aspiration (TBNA) in the diagnosis of mediastinal masses is currently a disputed subject. Previous studies have demonstrated that EBUS-TBNA performs better compared to conventional TBNA as it is capable of sampling in real-time compared with conventional TBNA. However, some clinicians consider conventional TBNA to be sufficient for diagnosis. In this meta-analysis, we evaluated these two methods according to diagnostic yield. A search was conducted through PubMed, Embase and the proceedings of major conferences, for studies comparing TBNA with EBUS-TBNA in the diagnosis of mediastinal masses. The identified studies were evaluated for publication bias and heterogeneity. The primary outcome was diagnostic yield. Pooled odds ratio (OR) estimated with $95 \%$ confidence intervals (CIs) was calculated using the fixed-effects model. Five studies, including a total of 407 patients, were included in the meta-analysis. The EBUS-TBNA arm was associated with a significantly higher OR compared to that of the TBNA arm in terms of diagnostic yield (OR=2.72, 95\% CI: 1.72-4.30, $\mathrm{P}<0.001)$. There was no evidence of heterogeneity $\left(\mathrm{I}^{2}=0 \%\right.$, $\mathrm{P}=0.540$ ) or publication bias (Egger's test, $\mathrm{P}=0.568$; Begg-Mazumdar test, $\mathrm{P}=0.806$ ). In conclusion, EBUS-TBNA and conventional TBNA are safe and provide good diagnostic yield in the diagnosis of hilar and mediastinal masses and
\end{abstract}

Correspondence to: Professor Chong Bai, Department of Respiratory Medicine, Changhai Hospital, Second Military Medical University, 168 Changhai Road, Shanghai 200433, P.R. China

E-mail: bc7878@sohu.com

${ }^{*}$ Contributed equally

Key words: mediastinal mass, transbronchial needle aspiration, endobronchial ultrasound guided-transbronchial needle aspiration, diagnostic yield, meta-analysis lymphadenopathies; however, EBUS-TBNA performs better compared to conventional TBNA, with a shorter aspiration time and higher sensitivity.

\section{Introduction}

Clinically, mediastinal masses are currently a common occurrence. The incidence of certain diseases, such as lung cancer and sarcoidosis, is on the increase. Accurate and early diagnosis of such diseases is crucial for the appropriate treatment and prognosis $(1,2)$. A major concern for clinicians worldwide is the establishment of a diagnostic method that is fast, accurate and minimally invasive.

Imaging is crucial in the diagnosis of mediastinal masses. An imaging scan may provide critical information with regard to the location, size and periphery of the lesions and it is fast and non-invasive. Positron emission tomography-computed tomography (PET-CT) may also indicate whether the mass is benign or malignant. However, more precise information is required for staging and typing.

Although mediastinoscopy or surgery is considered to be the gold standard for diagnosis (3), some patients are unable to tolerate the procedural injury and intravenous anesthesia. Therefore, efforts are focused on establishing a method that is easy to use, minimally invasive and efficient in providing the critical information required.

Transbronchial biopsy is such a method, particularly transbronchial needle aspiration (TBNA), which aspirates masses and pathological lymph nodes through an air tube, with minimal invasion (4). However, conventional TBNA requires the assistance of X-ray, CT or PET-CT; therefore, there remains the need for equipment that may guide real-time sampling. The development of the ultrasound technique enabled clinicians to perform esophageal endoscopic ultrasound-guided fine-needle aspiration (EUS-FNA) in the 1980s. Although this technique was efficient in real-time aspiration of para-esophageal masses, its range was limited for mediastinal masses (5) and the technique was developed further over the subsequent decades. When endobronchial ultrasound-guided (EBUS)-TBNA was introduced, it enabled sampling within a broader range $(6,7)$. 
Furthermore, there is continuous improvement, such as ventilation (8) and linear probes (9), which rendered this procedure easier, faster and more accurate. Therefore, EBUS-TBNA may be the optimal method. However, the equipment is costly and may be unaffordable for some hospitals. Furthermore, certain clinicians consider conventional TBNA sufficient for diagnosis, provided it is performed by a skilled expert. Over the last few years, particularly from 2000 onwards, several studies have focused on this issue. Certain studies indicated that EBUS-TBNA performs better in terms of diagnostic sensitivity and specificity, as well as safety $(10,11)$. However, other studies suggested that conventional TBNA was not inferior to EBUS-TBNA $(12,13)$. Although there have been several studies comparing these two methods, they all presented with drawbacks, particularly their small sample size. Therefore, we conducted this meta-analysis using the existing resources to compare the diagnostic yield of the two methods.

\section{Materials and methods}

Search strategy. A comprehensive literature search was conducted in order to identify studies comparing the diagnostic performance of EBUS-TBNA to that of TBNA in the detection of mediastinal masses. The eligible studies were required to use histopathological and/or cytological analysis, or close clinical follow-up for $\geq 6$ months after mediastinoscopy or surgery, as the reference standard. Studies that investigated EBUS-TBNA or conventional TBNA alone, studies that did not provide results on (or sufficient data to calculate) diagnostic yield and studies that assessed diagnostic yield only in metastatic mediastinal lymph nodes, were excluded.

A search was conducted through the electronic databases PubMed (from 1966 to April, 2011), Embase (from 1980 to April, 2011) and the Cochrane Library. The search terms included 'endobronchial ultrasound', 'endoscopic ultrasound' or 'endosonography', 'transbronchial ultrasound' and 'transbronchial needle aspiration'. We also reviewed the reference lists of original and review articles to identify relevant studies. There were no restrictions regarding publication language.

Study selection and data collection. Two reviewers (Z.T.Y. and Z.X.J.) independently assessed the eligibility of the identified studies and discrepancies were resolved by consensus. Data was collected using standardized data collection forms. The following information was extracted from each eligible study: first author's name, year of publication, description of study population, study design (prospective, retrospective or unknown), patient enrollment (consecutive or not), interpretation of test results (blinded or not), patient selection on the basis of CT- or PET-positive results or not, reference standard and diagnostic yield results.

A total of 991 citations were initially identified. After reviewing the titles and abstracts, 957 citations, such as case reports, reviews, reader letters and studies not assessing both methods, were excluded. After reading the full text, 5 studies were selected for inclusion in the planned meta-analysis (Fig. 1 and Table I) (12-16).

Statistical analysis. To calculate the pooled odds ratio (OR), the number of true positives in each arm was extracted from each

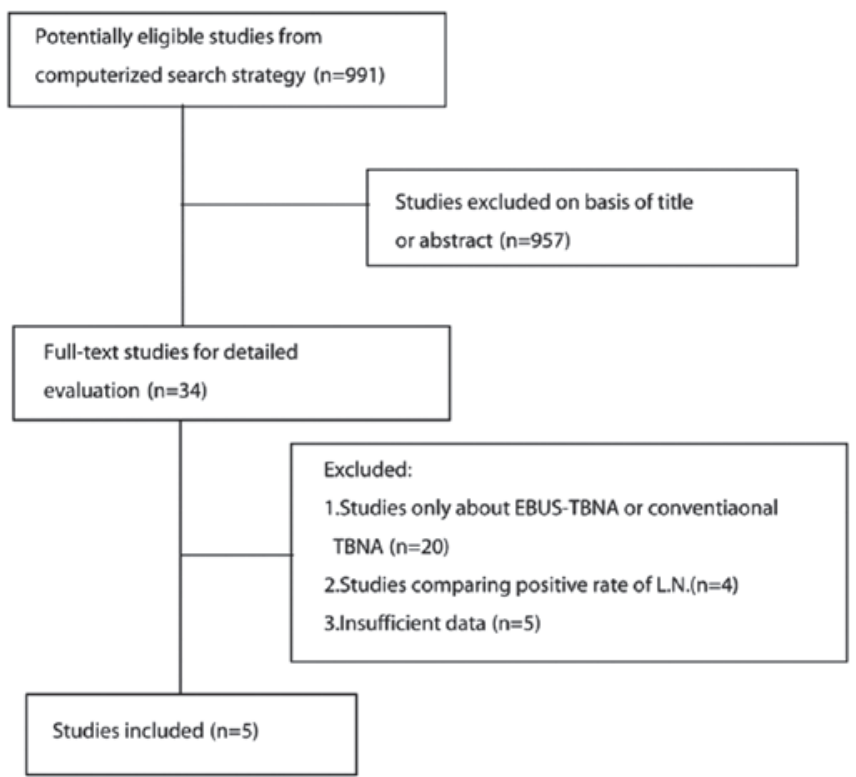

Figure 1. Process of eligible study identification. L.N., lymph node.

study and combined using the method described by Mantel and Haenszel (17). A pooled $\mathrm{OR}>1$ indicated a diagnostic yield in the EBUS-TBNA arm. Heterogeneity between the trials was evaluated by the $\chi^{2}$ test and $\mathrm{I}^{2}$ statistic (18). These indices were used to assess the percentage of variability across studies attributable to heterogeneity rather than chance. Statistical heterogeneity was considered significant when $\mathrm{P}<0.10$ for the $\chi^{2}$ test or $\mathrm{I}^{2}>50 \%$. When there was no statistically significant heterogeneity, a pooled effect was calculated with a fixed-effects model; otherwise, a random-effects model was employed. We also assessed the probability of publication bias according to the Egger's (19) and Begg-Mazumdar (20) tests. All reported $\mathrm{P}$-values were two-sided and $\mathrm{P}<0.05$ was considered to indicate a statistically significant difference. Statistical analyses were performed using RevMan software, version 5.0 (The Cochrane Collaboration).

\section{Results}

Study selection. The literature search yielded 991 studies, of which 957 were excluded after reviewing the title and abstract. The remaining 34 potentially eligible studies underwent a full-text review. However, 20 studies only focused on EBUS-TBNA or conventional TBNA, 4 studies compared the positive lymph node rates and 5 studies did not provide sufficient data. Ultimately, 5 studies (12-16), including a total of 407 patients, fulfilled all the inclusion criteria and were selected for the meta-analysis.

Characteristics of individual studies. Arslan et al (12) conducted a prospective study, enrolling 60 patients who were blindly randomized. The overall diagnostic yield was $66.7 \%(20 / 30)$ in the EBUS-TBNA and $33.33 \%(10 / 30)$ in the conventional TBNA group. The difference between the groups was statistically significant, particularly during the early stages of the disease. In the study conducted by Tremblay et al (13), EBUS-TBNA and TBNA were compared 
Table I. Characteristics of the trials included in the meta-analysis.

\begin{tabular}{|c|c|c|c|c|c|c|c|}
\hline Authors (refs.) & $\begin{array}{l}\text { Study } \\
\text { time }\end{array}$ & $\begin{array}{l}\text { Sample } \\
\text { size }\end{array}$ & $\begin{array}{l}\text { Mean } \\
\text { age }\end{array}$ & $\begin{array}{l}\text { Study } \\
\text { design }\end{array}$ & ROSE & $\begin{array}{l}\text { Conventional } \\
\text { TBNA }(\%)\end{array}$ & $\begin{array}{c}\text { EBUS- } \\
\text { TBNA }(\%)\end{array}$ \\
\hline Arslan et al (12) & 2006.7-2007.10 & 60 & 56.15 & Prospective, randomized & No & $10 / 30(33.3)$ & $20 / 30(66.7)$ \\
\hline Tremblay et al (13) & 2006.9-2007.08 & 50 & 40.2 & Randomized, blinded & No & $14 / 26(53.8)$ & $20 / 24(83.3)$ \\
\hline Herth et al (14) & 2001.6-2002.3 & 200 & 51.9 & Randomized & No & $66 / 100(66)$ & $85 / 100(85)$ \\
\hline Oki et al (15) & - & 15 & 55.2 & Prospective & No & $13 / 15(93)$ & $13 / 15(93)$ \\
\hline Shannon et al (16) & 1993.1-1994.11 & 82 & 60 & Prospective, randomized & Yes & $32 / 42(76.2)$ & $33 / 40(82.5)$ \\
\hline
\end{tabular}

ROSE, rapid on-site evaluation; TBNA, transbronchial needle aspiration; EBUS, endobronchial ultrasound.

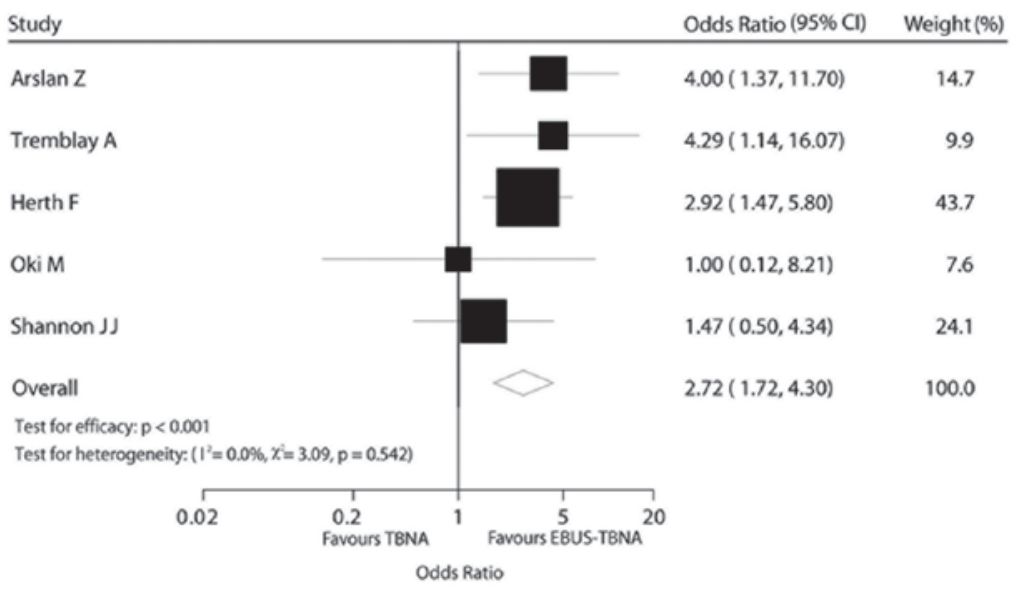

Figure 2. Comparison of diagnostic yield between transbronchial needle aspiration (TBNA) and endobronchial ultrasound (EBUS)-TBNA.

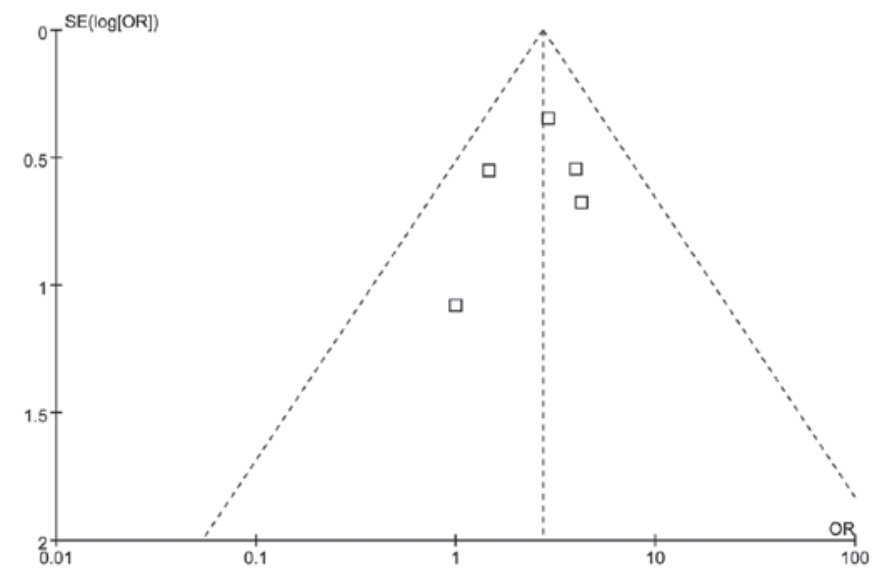

Figure 3. Funnel plot for diagnostic yield.

in 50 patients, randomly and blindly. EBUS-TBNA accurately diagnosed $20(83.30 \%)$ of the 24 patients and conventional TBNA diagnosed $14(53.80 \%)$ of the 26 patients. Furthermore, EBUS exhibited a higher aspiration efficiency (4-2.2 on average). Herth et al (14) conducted a large-scale study, including 200 randomized patients. The diagnostic outcome was $85 \%$ (EBUS-TBNA) vs. $66 \%$ (conventional TBNA). The patients in that study were also stratified by the location of the lymph nodes and the authors concluded that, when the lesion was in the subcarinal region, there was no difference between
EBUS-TBNA and conventional TBNA; however, when the lesion was located elsewhere, the difference was statistically significant. The 3 previously mentioned studies consistently confirmed that the diagnostic yield of EBUS-TBNA was higher compared with that of conventional TBNA; however, certain researchers were not convinced. In a study conducted by Oki et al (15), 15 patients were evaluated by EBUS-TBNA and conventional TBNA and the diagnostic yield was identical (93\% in both methods). Furthermore, a study conducted by Shannon et al (16) enrolled 82 patients. Rapid on-site evaluation (ROSE) by cytopathological examination was applied and the results suggested that there was no significant difference, with or without the use of ultrasound (Table I).

Patients, diagnostic methods and effect on diagnostic yield. All studies used an ultrasound bronchoscope provided by Olympus (Tokyo, Japan). The median number of participants was 77.5 (range, 15-200) and the median age was 53.5 years (range, 40-60 years). The diagnosis was based on histopathological and/or cytological analysis in all 5 studies. When the final diagnosis was not definitive, other diagnostic methods, such as clinical follow-up for $\geq 6$ months, mediastinoscopy or surgery were used.

The effect of the diagnostic method on diagnostic yield was estimated directly for all 5 trials. The EBUS-TBNA arm was associated with a significantly higher OR compared to that of the TBNA arm in terms of diagnostic yield [OR=2.72, 
95\% confidence interval (CI): 1.72-4.30, P<0.001; Fig. 2]. There was no evidence of heterogeneity among individual trials $\left(\mathrm{I}^{2}=0 \%, \mathrm{P}=0.540\right)$. We detected no evidence of publication bias using the Egger's $(\mathrm{P}=0.568)$ and the Begg-Mazumdar ( $\mathrm{P}=0.806$ ) tests (Fig. 2).

\section{Discussion}

Accurate staging enables the selection of the appropriate treatment, which may achieve higher survival rates and improve the quality of life of the patients. In our clinical practice, the diagnostic methods may be classified into invasive and non-invasive. Although mediastinal lymph nodes and masses may be apparent on CT or PET-CT, accurate diagnosis requires a tissue biopsy. The treatment options depend largely on histology and staging. Therefore, the invasive techniques are necessary. In order to determine the pathological type, tissue may be obtained under X-ray or CT guidance. Fiberoptic bronchoscopy or mediastinoscopy may be used. If a diagnosis cannot be confirmed with these methods, surgery may be the final option. Among these invasive methods, we always to select the least invasive procedure with the highest level of diagnostic sensitivity.

In a meta-analysis conducted by Holty et al (21), TBNA exhibited a relatively higher specificity (99\%), although the sensitivity was low (39\%), with a pooled major complication rate of $0.3 \%$. A meta-analysis by Micames et al (22) reported that EUS-FNA identified $83 \%$ of the patients (95\% CI: $0.78-0.87$ ) with positive and $97 \%$ of the patients (95\% CI: 0.96-0.98) with negative mediastinal lymph nodes. In a meta-analysis by Gu et al (1), the primary endpoint was the accuracy of EBUS-TBNA in detecting lymph node metastases in lung cancer. The results demonstrated that EBUS-TBNA exhibited the highest sensitivity (93\%, 95\% CI: 0.91-0.94) and specificity (100\%, 95\% CI: 0.99-1.000) (Figs. 2 and 3).

To the best of our knowledge, this meta-analysis was the first to compare the diagnostic yield between EBUS-TBNA and conventional TBNA. Our results demonstrated that EBUS-TBNA performed better compared with conventional TBNA and were consistent with those of well-designed previous studies $(13,15)$. Shannon et al $(16)$ reported that the ROSE method they used improved the diagnostic accuracy, which was consistent with the results of Chin et al (23); however, it requires costly and sophisticated equipment. In the studies of Herth et al (14) and Arslan et al (12), the patients were stratified according to the anatomic location of the pathological lymph nodes. The authors reported that EBUS-TBNA performed better in sites other than the subcarinal space. When the lesion was peripherally located, although EBUS-TBNA sampled more accurately and easily, the diagnostic yield was low and was improved via combination with other equipment $(24,25)$. Those findings suggest that when EBUS-TBNA is used for diagnostic purposes, the site of the lesion should be carefully considered. In our meta-analysis, no complication (such as, vessel injury) was recorded among the 407 patients. Rong et al (26)mentioned in his study that there were more complications in the conventional TBNA group, although the difference between EBUS-TBNA and TBNA was not statistically significant. According to our experience, if clinicians are familiarized with the anatomy, have mastered the technique and properly evaluate the patient's condition prior to the procedure, the majority of complications may be avoided.

There were certain limitations to our analysis, although our findings demonstrated that EBUS-TBNA performed better compared to conventional TBNA. The design and sample size of the enrolled studies did not meet our expectations. Other factors, such as the experience of the operators (27), the time of the aspiration and the location, size and nature of the lesions, may affect the final result. When these factors are well-controlled, the comparison may be of greater value.

In conclusion, EBUS-TBNA and conventional TBNA are safe and provide good diagnostic yield in the diagnosis of hilar and mediastinal masses and lymphadenopathies. However, EBUS-TBNA performs better compared with conventional TBNA, with shorter aspiration time and higher sensitivity. The result may be further improved via combination with ROSE. However, further randomized, multicenter clinical trials including larger sample sizes are required to further establish the advanatages of EBUS-TBNA over conventional TBNA in the diagnosis of mediastinal masses.

\section{Acknowledgements}

This study was supported by grants from the National Natural Science Foundation of China (nos. 30871133 and 81270073).

\section{References}

1. Gu P, Zhao YZ, Jiang LY, Zhang W, Xin Y and Han BH: Endobronchial ultrasound-guided transbronchial needle aspiration for staging of lung cancer: a systematic review and meta-analysis. Eur J Cancer 45: 1389-1396, 2009.

2. Varela-Lema L, Fernandez-Villar A and Ruano-Ravina A: Effectiveness and safety of endobronchial ultrasound-transbronchial needle aspiration: a systematic review. Eur Respir J 33: 1156-1164, 2009.

3. Rusch VW: Mediastinoscopy: an endangered species? J Clin Oncol 23: 8283-8285, 2005

4. Arroliga AC and Matthay RA: The role of bronchoscopy in lung cancer. Clin Chest Med 14: 87-98, 1993.

5. Annema JT, Versteegh MI, Veselic M, Voigt P and Rabe KF: Endoscopic ultrasound-guided fine-needle aspiration in the diagnosis and staging of lung cancer and its impact on surgical staging. J Clin Oncol 23: 8357-8361, 2005.

6. Silvestri GA, Hoffman BJ, Bhutani MS, et al: Endoscopic ultrasound with fine-needle aspiration in the diagnosis and staging of lung cancer. Ann Thorac Surg 61: 1441-1446, 1996.

7. Hurter T and Hanrath P: Endobronchial sonography: feasibility and preliminary results. Thorax 47: 565-567, 1992.

8. Becker HD: EBUS: a new dimension in bronchoscopy. Of sounds and images - a paradigm of innovation. Respiration 73: 583-586, 2006.

9. Herth FJ and Eberhardt R: Actual role of endobronchial ultrasound (EBUS). Eur Radiol 17: 1806-1812, 2007.

10. Cetinkaya E, Gunluoglu G, Ozgul A, et al: Value of real-time endobronchial ultrasound-guided transbronchial needle aspiration. Ann Thorac Med 6: 77-81, 2011.

11. Ost DE, Ernst A, Lei X, et al: AQuIRE Bronchoscopy Registry: Diagnostic yield of endobronchial ultrasound-guided transbronchial needle aspiration: results of the AQuIRE Bronchoscopy Registry. Chest 140: 1557-1566, 2011.

12. Arslan Z, Ilgazli A, Bakir M, Yildiz K and Topçu S: Conventional vs. endobronchial ultrasound-guided transbronchial needle aspiration in the diagnosis of mediastinal lymphadenopathies. Tuberk Toraks 59: 153-157, 2011.

13. Tremblay A, Stather DR, Maceachern P, Khalil M and Field SK: A randomized controlled trial of standard vs endobronchial ultrasonography-guided transbronchial needle aspiration in patients with suspected sarcoidosis. Chest 136: 340-346, 2009.

14. Herth F, Becker HD and Ernst A: Conventional vs. endobronchial ultrasound-guided transbronchial needle aspiration: a randomized trial. Chest 125: 322-325, 2004. 
15. Oki M, Saka H, Kitagawa C, Tanaka S, Shimokata T, Kawata Y, Mori K, Kajikawa S, Ichihara S and Moritani S: Real-time endobronchial ultrasound-guided transbronchial needle aspiration is useful for diagnosing sarcoidosis. Respirology 12: 863-868, 2007.

16. Shannon JJ, Bude RO, Orens JB, et al: Endobronchial ultrasoundguided needle aspiration of mediastinal adenopathy. Am J Respir Crit Care Med 153: 1424-1430, 1996.

17. Deeks JJ, Higgins JPT and Altman DG: Analysing data and undertaking meta-analyses. In: Cochrane Handbook for Systematic Reviews of Interventions. Higgins JPT and Green S (eds). Version 5.0.0 (updated February 2008). Available from url: http://www.mrc-bsu.cam.ac.uk/cochrane/handbook500/. Accessed July 15, 2011.

18. Higgins JP, Thompson SG, Deeks JJ and Altman DG: Measuring inconsistency in meta-analyses. BMJ 327: 557-560, 2003

19. Egger M, Davey Smith G, Schneider M and Minder C: Bias in meta-analysis detected by a simple, graphical test. BMJ 315: 629-634, 1997

20. Begg CB and Mazumdar M: Operating characteristics of a rank correlation test for publication bias. Biometrics 50: 1088-1101, 1994.

21. Holty JE, Kuschner WG and Gould MK: Accuracy of transbronchial needle aspiration for mediastinal staging of non-small cell lung cancer: a meta-analysis. Thorax 60: 949-955, 2005.
22. Micames CG, McCrory DC, Pavey DA, Jowell PS and Gress FG: Endoscopic ultrasound-guided fine-needle aspiration for non-small cell lung cancer staging: A systematic review and metaanalysis. Chest 131: 539-548, 2007.

23. Chin R Jr, McCain TW, Lucia MA, et al: Transbronchial needle aspiration in diagnosing and staging lung cancer: how many aspirates are needed? Am J Respir Crit Care Med 166: 377-381, 2002.

24. Sheski FD and Mathur PN: Endobronchial ultrasound. Chest 133 264-270, 2008

25. Vilmann P and Puri R: The complete 'medical' mediastinoscopy (EUS-FNA + EBUS-TBNA). Minerva Med 98: 331-338, 2007.

26. Rong F, Xiao SH, Liu J, Li YX, Mai HY and Lu YS. A comparative research on the conventional transbronchial needle aspiration and endobronchial ultrasound-guided transbronchial needle aspiration for the diagnosis of mediastinum lesions. Zhonghua Jie He He Hu Xi Za Zhi 34: 120-122, 2011.

27. Gasparini S, Zuccatosta L and De Nictolis M: Transbronchial needle aspiration of mediastinal lesions. Monaldi Arch Chest Dis 55: $29-32,2000$ 